\title{
CdZnTe spectra improvement through digital pulse amplitude correction using the linear sliding method
}

\author{
João M. Cardoso*, J. Basílio Simões, Tiago Menezes, Carlos M.B.A. Correia \\ Electronics and Instrumentation Group, Department of Physics, University of Coimbra, Coimbra 3004-516, Portugal
}

\begin{abstract}
This paper presents innovative algorithms for optimum filtering, rise-time discrimination and proper correction of solid-state detector pulses based on digital pulse processing (DPP) techniques and on a Linear Sliding Method. These techniques allow a significant improvement on the spectral resolution by taking into account both the electronic noise and the pulse rise time fluctuations mainly due to the incomplete charge collection in the CZT crystal. In order to develop these algorithms pre-amplified pulses coming from a planar XR100-T detector (Amptek) were digitized by a 12bit PCI acquisition card $(125 \mathrm{MHz})$ hosted by a PC platform. The presented results show that, due to the possibility of fine adjustments on the discrimination and correction parameters during the processing stage, this flexible DPP implementation leads to significantly better results than the analog hardware discrimination solution. Comparative analysis of Am-241 spectra show a significant reduction of the low-energy "tail" on the $59.5 \mathrm{keV}$ photo-peak.
\end{abstract}

(C) 2003 Elsevier Science B.V. All rights reserved.

PACS: $07.05 . \mathrm{Hd} ; 84.30 . \mathrm{Sk}$; 07.50.Qx; 02.70.Hm

Keywords: Digital signal processing; Pulse processing algorithms; Data acquisition; Rise time discrimination; Spectral resolution

\section{Introduction}

The Digital Pulse Processing (DPP) approach in Nuclear Spectrometry is a fertile ground for the adaptation of traditional, and often cumbersome solutions adopted in analog systems, with significant additional advantages.

The traditional systems are often designed for general-purpose applications and therefore are somehow limited in their flexibility and performance. The use of these same spectroscopic systems based on digital techniques allows the

*Corresponding author. Tel.: + 351-23-941-0109; fax: + 35123-982-9158.

E-mail address: cardoso@lei.fis.uc.pt (J.M. Cardoso). implementation of a series of processing algorithms capable of attaining near-optimal performance. For long, it has been recognized, for instance, that the best energy resolution obtained with theoretically optimum signal-to-noise filters [1], can only be implemented through digital methods. The DPP solution has already been used in several processing platforms, both commercial and experimental, with many different technologic implementations, always with the determined purpose of achieving a faster, more accurate and versatile nuclear spectrometer [2].

The presented work was carried out in the sequence of previously developed DPP systems and architecture solutions [2-4], with the ultimate goal of developing a portable high-performance 
digital spectrometry system based on DSPs. In this way, it will be possible to apply optimized algorithms for pulse shaping, shape analysis, pileup correction, etc., and simultaneously assure a high-count rate through the use of a scalable parallel DSP architecture.

Having that goal in mind, and in the scope of this long-term work, one can demonstrate the versatility of this approach by implementing a simple, yet powerful way to reduce one of the major drawbacks of the CdZnTe detectors: the pulse rise-time fluctuations. In fact, the pulse discrimination and amplitude correction can be easily accomplished through the DPP technique avoiding the discard of the "slow rising" pulses, as often occur with the CdZnTe analog spectrometers. Using this method every detector pulses are processed and contribute to the final spectrum.

This document presents the overall architecture of the Digital Spectrometer used to develop the DPP algorithms (Section 2), followed by a brief explanation of the pulse shape processing algorithms (Section 3) with special relevance for the digital rise-time discrimination (Section 3.1) and amplitude correction (Section 3.2) methods.

\section{The digital spectrometer}

Considering the algorithm development purpose of this work, a commercial off-the-shelf digital spectrometer was assembled (Fig. 1). It is composed by a CdZnTe detector (XR-100 T model from Amptek using a $3 \times 3 \times 2 \mathrm{~mm}^{3}$ crystal), a signal conditioning module (impedance adjustment and interface), a digitizing platform (12-bit Signatec's PDA12A-512k PCI waveform digitizer hosted by a PC) and a set of $\mathrm{C}++$ pulse processing routines, developed in the scope of this work.

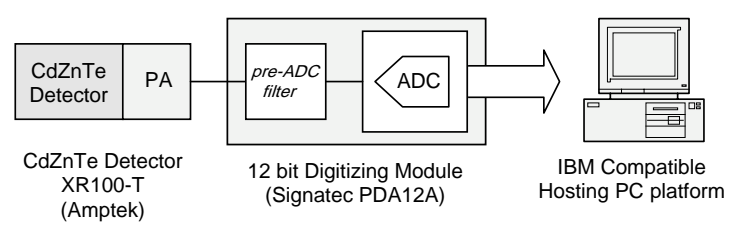

Fig. 1. Digital spectrometer architecture.
The basic operation mode consists on the full digitizing of the stream of pre-amplified pulses (internally triggered by the PDA12A module) with subsequent transferring to the PC memory and appropriate real time processing. The overall effect is similar to the traditional spectrometers apart of the lower throughput performance of this particular experimental setup.

\section{Digital pulse processing}

Further, we describe the DPP algorithms implemented in the nuclear spectrometer presented here (Table 1).

Each digitized pulse $\left(x_{\mathrm{n}}\right)$ is firstly numerically differentiated in order to determine its rising edge position (Eq. (1)). This procedure is used, along with the hardware trigger information, to give us an estimation of the region that should be used in the pulse amplitude determination. The amplitude is obtained through the convolution with a weighting function $\left(S_{\mathrm{n}}\right)$ (Eq. (2)) whose parameters are chosen according to the experimental characteristics of the spectrometer (noise sources effectively present on the detector-preamplifier, pulse rise-time, count rate, etc.). Just considering the signal-to-noise ratio, the best resolution is attained with the finite cusp weighting function $\left(w_{\mathrm{n}}\right)$ (Eq. (3)). The correspondent convolution maximum, that is proportional to the pulse amplitude, is then stored in order to obtain the energy spectrum (Fig. 2).

Pulse rise-time is also estimated through a simple counter routine and associated with the correspondent pulse amplitude.

Table 1

Basic DPP algorithm expressions

Step estimation $\max \left(y_{n}\right)=\max \sum_{i}\left(x_{n+i}-x_{n-i}\right)$

Convolution $\max \left(S_{n}\right) \rightarrow S_{n}=\frac{1}{N} \sum_{\tau} x_{n} w_{n-\tau}$

Weighting function $\quad w_{n}=-A \frac{\mathrm{d}}{\mathrm{d} n}\left(\mathrm{e}^{-n / \kappa}\right)$ 


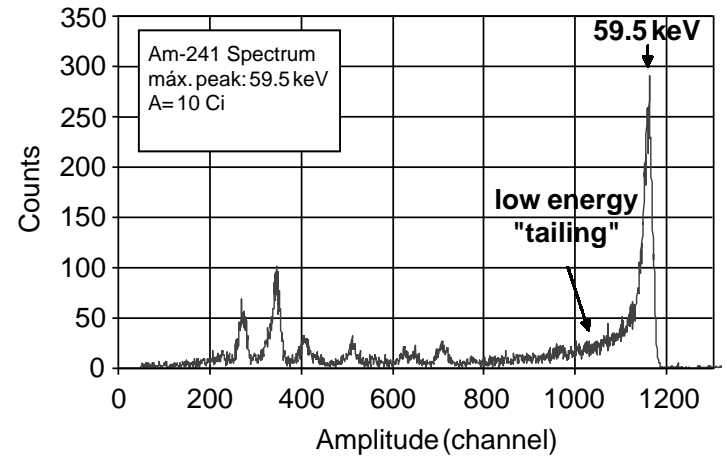

Fig. 2. Am-241 energy spectrum. Photo-peak tail due to pulse rise-time fluctuations is evident to degrade spectral resolution.

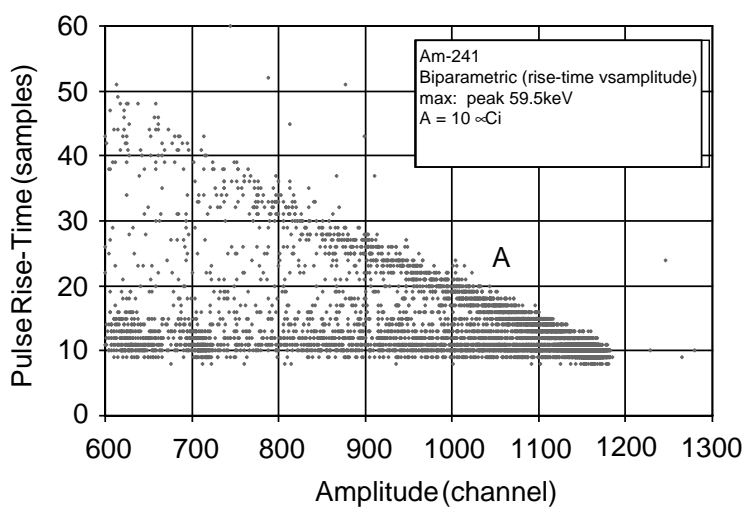

Fig. 3. Bi-parametric representation of pre-amplified pulses. 512 samples were taken for each pulse. A 64-point weighting function (truncated cusp) with 14-point top was used. Each dot represents one pulse.

The determination of both the amplitude and rise-time allows the bi-parametric representation of the pre-amplified pulses and the understanding of the real cause for the photo-peak low energy tail (Fig. 3).

\subsection{Digital rise-time discrimination}

The major responsible factor for the fluctuations in the pre-amplified pulse rise-time of the $\mathrm{CdZnTe}$ detector is the incomplete charge collection due to the presence of charge trapping centers in the crystal. This occurs mainly due to small imperfections in the crystalline structure of the detector (impurities, planar displacements, surface effects, etc.) that trap part of the charge produced by the incident radiation (from the cathode side) and thus prevents its full collection in the electrodes [5]. This collection efficiency is often described by the Hecht expression [6].

The incomplete charge collection is revealed on the final spectrum yield through the appearance of a low energy "tailing" in the photo-peaks, worsening the spectral resolution (Fig. 2) [7]. This is a well-known drawback of CZT detectors. Considering this effect, Amptek ${ }^{\circledR}$ introduced in the market an analog Rise-Time Discriminator (RTD) based on a charge comparison method [8]. However, this is not a standard discrimination module for most of the CZT detectors. It discards the "slow-rising" pulses but adjustment of the discrimination threshold is not possible.

On the contrary, using software driven DPP techniques, pulse discrimination based on rise-time criteria is quite straightforward. It simply requires comparison with an adjustable parameter (threshold). However, care must be taken in the choice of this threshold since excessive lowering can significantly reduce photo-peak efficiency. According to the measured bi-parametric distribution (Fig. 3), a threshold of 16 samples on the rise-time resulted in a good compromise between tail reduction and the $59.5 \mathrm{keV}$ peak yield. The resolution improvement reached $0.5 \%$ when compared with the original spectrum (Fig. 2), which is slightly better than the $0.3 \%$ improvement obtained with the analog RTD.

Another important issue that accounts for the improvement of spectral immunity to rise-time fluctuations is the weighting function shape. The application of a flat top function (truncated cusp) dramatically improved the spectrometer linearity. This is a well-known way of reducing the typical spectral offset due to ballistic deficit since the samples of the pulse leading edge are simply discarded. In our experimental setup weighting functions with a flat-top of 14 samples avoid the ballistic deficit characteristic spectrum shift.

\subsection{Amplitude correction — linear sliding method}

More than just performing a flexible rise-time discrimination, the major advantage of the DPP is 


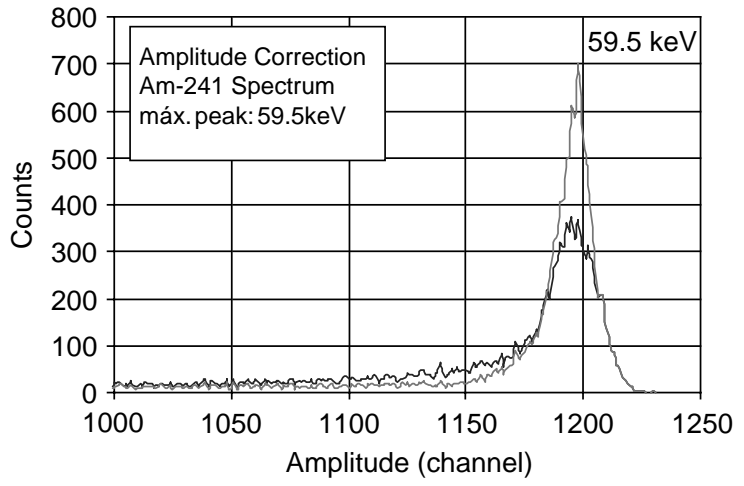

Fig. 4. Amplitude correction using Linear Sliding Method [9].

its ability to easily correct the consequent amplitude deficit. Careful analysis of the bi-parametric distribution (Fig. 3), reveals that the pulses responsible for the photo-peak tail occur in a relatively well-defined region (A). In this region, "slower" pulses have higher amplitude attenuations according to the charge trapping mechanism referred earlier. It is also worth mentioning that this amplitude to rise-time dependence is approximately linear, which also makes sense since the electric field $E$ in the CZT crystal is also approximately linear. Based on these considerations a simple amplitude correction algorithm was developed for the pulses above the discrimination threshold: the Linear Sliding Method.

The corrected amplitude is obtained through expression (4) using the amplitude, $a$, and the pulse rise-time, $t_{\text {rise-time, }}$ coming from the digital pulse processing algorithms, and the parameter $m$ that is obtained from an initial calibration procedure. ${ }^{1}$ This calibration procedure consists on the determination of the slope, $\mathrm{m}$, of the straight line best fitting the region $\mathrm{A}$ of the bi-parametric function represented in Fig. 3. So, every new acquired pulse will have its amplitude corrected accordingly to

$a_{\text {corrected }}=-\frac{t_{\text {rise-time }}-\text { am }}{m}$.

This procedure can be pictured as the linear "sliding" of the pulses along a straight line with the same slope of the linear fit. The overall effect on the

\footnotetext{
${ }^{1}$ It should be noticed that this calibration procedure is energy dependent.
}

final spectrum is clearly evident in Fig. 4 that compares both the corrected and the raw peaks.

This method can be considered as a particular case of a generic procedure usually applied to improve spectral resolution of planar semiconductor detectors over all the energetic dynamic range [10].

\section{Conclusions}

A versatile digital spectrometer based on offthe-shelf commercial modules was presented. It was shown that DPP can easily replace, with significant advantages, complex experimental setups that would be otherwise necessary to perform rise-time discrimination of CZT pulses. An amplitude correction algorithm (linear sliding method) was implemented with impressive spectral results from the photo-peak efficiency point of view.

\section{Acknowledgements}

Authors thank the travel and accommodation support provided by Fundação Calouste Gulbenkian.

\section{References}

[1] J. Basílio Simões, C.M.B.A. Correia, Nucl. Instr. and Meth. A 422 (1999) 405.

[2] J.M.R. Cardoso, J. Basílio Simões, C. Correia, Nucl. Instr. and Meth. A 422 (1998) 404.

[3] J.B. Simões, J. Cardoso, C. Correia, IEEE Conference Record of NSSMIC, 1999.

[4] B. Redus, Charge trapping in XR100 T detectors, Amptek application note, 2000, p. 14.

[5] L.A. Hamel, et al., Nucl. Instr. and Meth. A 380 (1996) 238.

[6] Y. Eisen, et al., Nucl. Instr. and Meth. A 353 (1994) 60.

[7] V. Jordanov, et al., Nucl. Instr. and Meth. A 380 (1996) 353.

[8] B. Keele, et al., IEEE Trans. Nucl. Sci. 43 (1996) 1365.

[9] P. Nicholson, Nuclear Electronics, Wiley, New York, 1974.

[10] J.M. Cardoso, Development of digital pulse processing algorithms for fast acquisition in nuclear spectrometry, Experimental Physics, M.Sc. Thesis, University of Coimbra, Portugal, 2001. 